\title{
"Status of macro nutrients in some soils of Central Farm of MKV, Parbhani (Maharashtra)"
}

\author{
S. L. Waikar, V. D. Patil and A. L. Dhamak \\ Department of Soil Science and Agricultural Chemistry, College of Agriculture, Vasantrao Naik Marathwada \\ Krishi Vidyapeeth, Parbhani -431402 (Maharashtra state) India
}

\begin{abstract}
The present investigation was carried out to study nutrients status of Central Farm - B - Block of $M K V$, Parbhani". Total one hundred surface soil samples were collected from Central Farm MKV, Parbhani. These soil samples were analyzed for physico-chemical properties and fertility status of soil. The data revealed that the soils of - B - block were neutral to alkaline in soil reaction, safe in electrical conductivity, low to high in organic carbon content and non-calcareous to calcareous in nature. According to the concept of "soil nutrient index" in general the status of available nitrogen, phosphorus and sulphur were "low". While "high" in content of available potassium, calcium, magnesium content in soils of B - block. Thus, it can be concluded that the physico-chemical properties like $\mathrm{pH}, \mathrm{EC}$, organic carbon and $\mathrm{CaCO}_{3}$ contents alone or in combination controls the availability of nutrients. Hence, build up of organic carbon status by different means is a practicable tool in our hands to minimize deficiency area of these soils with reference to nitrogen, phosphorus in these soils.
\end{abstract}

Key Words : Physico-chemical properties, Fertility and Macro nutrients

\section{Introduction}

In Maharashtra, most of the soils have developed over basaltic parent materials and are heavier in texture and productivity. The parent material is the main source of many plant nutrients. These determines the physical properties of soil such as texture, structure, water holding capacity and improve nutrient availability in the soils. Parbhani belongs to the Godavari peninsular region situated on the northern side of Marathwada. The northern part of district is highly and mountainous having excessive relief. The district is covered by basaltic lava flow. The climate of the district is sub-tropical, monsoonic type with mild winter and hot summer. Soil fertility is one of the important factor contributing yields of the crops. The major and minor nutrients govern the fertility of the soil and control the yields of the crops. Soil characterization in relation to evaluation of fertility status of the soils of an area or region is an important aspect in context of sustainable agricultural production. Because of imbalanced and inadequate fertilizer use coupled with low efficiency of other inputs, the response (production) efficiency of other chemical fertilizer nutrients has declined tremendously under intensive agriculture in recent year (Meena et al.,2006). The information with respect to physico-chemical properties and availability of macro nutrients of the study area was lacking. Therefore, the present investigation was planned.

\section{Material And Methods.}

Collection of soil samples: one hundred surface soil samples were collected, completely air dried, passed through $2 \mathrm{~mm}$ sieve and stored in properly labeled plastic bags. The soil $\mathrm{pH}, \mathrm{EC}$, Organic Carbon, available K, Exchangeable $\mathrm{Ca}, \mathrm{Mg}$ were estimated by the standard procedures as described by Jackson (1973). The available $\mathrm{N}$ was analyzed by using alkaline potassium permanganate (Subbaiah and Asija, 1956). Available S was determined by using $0.15 \% \mathrm{CaCl}_{2}$ solution.

\section{Result And Discussion}

The results of study presented in Table1 and fig. 1 indicated that all the soil samples of Central Farm B - Block of MKV, Parbhani were neutral $(87 \%)$ and alkaline $(13 \%)$ in soil reaction, safe in electrical conductivity (100\%), low (35\%),medium(46\%) and high (19\%) in organic carbon content and soils were noncalcareous $(19 \%)$ and calcareous $(81 \%)$ in nature. The $\mathrm{pH}$ of soil samples varied from 7.03 to 8.01 in Central Farm - B - Block of MKV, Parbhani . It may be due to formation of these soils from basaltic parent material rich in basic cations. Similar results were reported by Padole and Mahajan (2003). EC of soil samples varied from 0.10 to $0.75 \mathrm{dSm}^{-1}$ in Central Farm - B - Block of MKV, Parbhani. The organic carbon content was varied from 1.1 to $9.3 \mathrm{~g} \mathrm{~kg}^{-1}$ in Central Farm - B - Block of MKV, Parbhani. It indicates that majority of these soils were low to high in organic carbon content. The high content of organic carbon might be due to addition of organic matter through either artificially or naturally and its subsequent decomposition. These results was confirmatory with results reported by More et al. (2013). The free $\mathrm{CaCO}_{3}$ content was varied from 2.0 to $108 \mathrm{~g}$ 
$\mathrm{kg}^{-1}$ in Central Farm - B - Block of MKV, Parbhani indicating non-calcareous to calcareous in nature of these soils.

Table No 1. Chemical properties of soils of central farm - B - block of MKV, Parbhani

\begin{tabular}{|c|c|c|c|c|c|}
\hline SR.NO & Block No & $\mathrm{pH}$ & $\begin{array}{c}\mathrm{EC} \\
\left(\mathrm{dSm}^{-1}\right)\end{array}$ & $\begin{array}{c}\text { Organic carbon } \\
\left(\mathrm{g} \mathrm{kg}^{-1}\right)\end{array}$ & $\begin{array}{l}\mathrm{CaCO} 3 \\
\left(\mathrm{~g} \mathrm{~kg}^{-1}\right) \\
\end{array}$ \\
\hline 1 & 29 & $\begin{array}{c}7.67-7.73 \\
(7.70)\end{array}$ & $\begin{array}{c}0.25-0.36 \\
(0.31)\end{array}$ & $\begin{array}{l}5.2-6.6 \\
(5.90)\end{array}$ & $\begin{array}{c}62.0-79.0 \\
(68.3)\end{array}$ \\
\hline 2 & 27 & $\begin{array}{c}7.73-7.86 \\
(7.79)\end{array}$ & $\begin{array}{c}0.27-0.41 \\
(0.34)\end{array}$ & $\begin{array}{c}7.8-8.2 \\
(8.0)\end{array}$ & $\begin{array}{c}74.0-84.0 \\
(79.00)\end{array}$ \\
\hline 3 & 22 & $\begin{array}{c}7.75-7.83 \\
(7.78)\end{array}$ & $\begin{array}{c}0.32-0.41 \\
(0.36)\end{array}$ & $\begin{array}{l}5.6-7.9 \\
(6.60)\end{array}$ & $\begin{array}{c}52.0-78.0 \\
(69.2)\end{array}$ \\
\hline 4 & 23 & $\begin{array}{c}7.71-7.79 \\
(7.75) \\
\end{array}$ & $\begin{array}{c}0.3-0.39 \\
(0.34) \\
\end{array}$ & $\begin{array}{c}4.8-8.5 \\
(6.3) \\
\end{array}$ & $\begin{array}{c}38.0-88.0 \\
(65.2) \\
\end{array}$ \\
\hline 5 & 25 & $\begin{array}{c}7.74-7.75 \\
(7.74)\end{array}$ & $\begin{array}{c}0.33-0.37 \\
(0.34)\end{array}$ & $\begin{array}{c}6.6-6.8 \\
(6.7)\end{array}$ & $\begin{array}{c}50.0-69.0 \\
(60.0)\end{array}$ \\
\hline 6 & 26 & $\begin{array}{c}7.71-7.74 \\
(7.72) \\
\end{array}$ & $\begin{array}{c}0.31-0.32 \\
(0.31) \\
\end{array}$ & $\begin{array}{c}4.1-8.1 \\
(6.1) \\
\end{array}$ & $\begin{array}{c}62.0-64.0 \\
(63.0) \\
\end{array}$ \\
\hline 7 & 28 & $\begin{array}{c}7.12-7.77 \\
(7.47) \\
\end{array}$ & $\begin{array}{c}0.35-0.73 \\
(0.51) \\
\end{array}$ & $\begin{array}{c}1.1-7.4 \\
(3.8)\end{array}$ & $\begin{array}{c}58.0-78.0 \\
(67.8) \\
\end{array}$ \\
\hline 8 & 30 & $\begin{array}{c}7.15-7.5 \\
(7.30)\end{array}$ & $\begin{array}{c}0.59-0.72 \\
(0.65)\end{array}$ & $\begin{array}{c}7.4-7.9 \\
(7.7)\end{array}$ & $\begin{array}{c}42.0-80.0 \\
(62.0)\end{array}$ \\
\hline 9 & 21 & $\begin{array}{c}7.66-7.92 \\
(7.78)\end{array}$ & $\begin{array}{c}0.45-0.75 \\
(0.56)\end{array}$ & $\begin{array}{c}4.5-5.9 \\
(5.0)\end{array}$ & $\begin{array}{c}49.0-82.0 \\
(61.0)\end{array}$ \\
\hline 10 & $22 \mathrm{SB}$ & $\begin{array}{l}7.4-7.7 \\
(7.61)\end{array}$ & $\begin{array}{c}0.35-0.52 \\
(0.42)\end{array}$ & $\begin{array}{c}4.1-6.6 \\
(5.6)\end{array}$ & $\begin{array}{c}28.0-80.0 \\
(47.6)\end{array}$ \\
\hline 11 & $23 \mathrm{SB}$ & $\begin{array}{c}7.24-7.93 \\
(7.74)\end{array}$ & $\begin{array}{c}0.41-0.66 \\
(0.49)\end{array}$ & $\begin{array}{c}1.1-9.3 \\
(5.9)\end{array}$ & $\begin{array}{c}44.0-89.0 \\
(68.0)\end{array}$ \\
\hline 12 & $24 \mathrm{SB}$ & $\begin{array}{c}7.75-7.86 \\
(7.79)\end{array}$ & $\begin{array}{c}0.47-0.51 \\
(0.48)\end{array}$ & $\begin{array}{c}4.5-6.7 \\
(5.8)\end{array}$ & $\begin{array}{c}34.0-64.0 \\
(49.5)\end{array}$ \\
\hline 13 & 24 & $\begin{array}{c}7.61-7.92 \\
(7.73) \\
\end{array}$ & $\begin{array}{c}0.3-0.71 \\
(0.48) \\
\end{array}$ & $\begin{array}{c}2.4-7.8 \\
(4.7) \\
\end{array}$ & $\begin{array}{c}32.0-72.0 \\
(50.6) \\
\end{array}$ \\
\hline 14 & $27 \mathrm{SB}$ & $\begin{array}{c}7.76-7.90 \\
(7.84)\end{array}$ & $\begin{array}{c}0.29-0.40 \\
(0.35)\end{array}$ & $\begin{array}{c}2.7-8.8 \\
(5.7)\end{array}$ & $\begin{array}{c}63.0-89.0 \\
(75.4)\end{array}$ \\
\hline 15 & $25 / 26 \mathrm{SB}$ & $\begin{array}{c}7.81-7.92 \\
(7.85) \\
\end{array}$ & $\begin{array}{c}0.32-0.54 \\
(0.39) \\
\end{array}$ & $\begin{array}{c}1.7-9.0 \\
(6.0) \\
\end{array}$ & $\begin{array}{c}42.0-99.0 \\
(67.7) \\
\end{array}$ \\
\hline 16 & 8 & $\begin{array}{c}7.85-7.91 \\
(7.87) \\
\end{array}$ & $\begin{array}{c}0.28-0.40 \\
(0.35) \\
\end{array}$ & $\begin{array}{c}4.1-8.5 \\
(6.1) \\
\end{array}$ & $\begin{array}{c}30.0-56.0 \\
(41.6) \\
\end{array}$ \\
\hline 17 & 9 & $\begin{array}{c}7.21-7.96 \\
(7.65)\end{array}$ & $\begin{array}{c}0.27-0.41 \\
(0.33)\end{array}$ & $\begin{array}{c}2.4-8.5 \\
(5.9)\end{array}$ & $\begin{array}{c}29.0-82.0 \\
(55.0)\end{array}$ \\
\hline 18 & 10 & $\begin{array}{c}7.32-7.99 \\
(7.80)\end{array}$ & $\begin{array}{c}0.1-0.36 \\
(0.27)\end{array}$ & $\begin{array}{c}3.1-5.9 \\
(4.8)\end{array}$ & $\begin{array}{c}40.0-79.0 \\
(63.0)\end{array}$ \\
\hline 19 & 20 & $\begin{array}{c}7.15-7.90 \\
(7.48)\end{array}$ & $\begin{array}{c}0.26-0.37 \\
(0.32)\end{array}$ & $\begin{array}{c}1.7-7.2 \\
(4.2)\end{array}$ & $\begin{array}{c}42.0-68.0 \\
(55.5)\end{array}$ \\
\hline 20 & 16 & $\begin{array}{c}7.25-7.89 \\
(7.56) \\
\end{array}$ & $\begin{array}{c}0.25-0.41 \\
(0.33)\end{array}$ & $\begin{array}{c}3.1-7.8 \\
(5.9)\end{array}$ & $\begin{array}{c}61.0-108.0 \\
(90.5)\end{array}$ \\
\hline 21 & 37 & $\begin{array}{c}7.03-8.01 \\
(7.53) \\
\end{array}$ & $\begin{array}{c}0.3-0.43 \\
(0.37) \\
\end{array}$ & $\begin{array}{c}4.5-7.9 \\
(5.7) \\
\end{array}$ & $\begin{array}{c}62.0-83.0 \\
(73.0) \\
\end{array}$ \\
\hline 22 & 17 & $\begin{array}{c}7.62-8.01 \\
(7.90)\end{array}$ & $\begin{array}{c}0.35-0.42 \\
(0.38)\end{array}$ & $\begin{array}{c}2.2-6.0 \\
(3.9)\end{array}$ & $\begin{array}{c}60.0-92.0 \\
(73.6)\end{array}$ \\
\hline 23 & 18 & $\begin{array}{c}7.89-7.97 \\
(7.91)\end{array}$ & $\begin{array}{c}0.28-0.37 \\
(0.33)\end{array}$ & $\begin{array}{c}3.1-7.2 \\
(4.7) \\
\end{array}$ & $\begin{array}{c}59.0-100.0 \\
(76.2) \\
\end{array}$ \\
\hline 24 & 19 & $\begin{array}{c}7.78-7.89 \\
(7.83) \\
\end{array}$ & $\begin{array}{c}0.36-0.37 \\
(0.36)\end{array}$ & $\begin{array}{c}7.2-9.3 \\
(8.2) \\
\end{array}$ & $\begin{array}{c}54.0-59.0 \\
(56.5) \\
\end{array}$ \\
\hline
\end{tabular}

(Figures in parenthesis indicates average value)

Table No: 2 Available Primary and Secondary Nutrient Status of Soils of central farm - B - block of MKV, Parbhani.

\begin{tabular}{|c|c|c|c|c|c|c|c|}
\hline SR.NO & $\begin{array}{c}\text { Block } \\
\text { No }\end{array}$ & $\begin{array}{c}\mathrm{N} \\
\left(\mathrm{kg} \mathrm{ha}^{-1}\right)\end{array}$ & $\begin{array}{c}\mathrm{P}_{2} \mathrm{O}_{5} \\
\left(\mathrm{~kg} \mathrm{ha}^{-1}\right)\end{array}$ & $\begin{array}{c}\mathrm{K}_{2} \mathrm{O} \\
\left(\mathrm{kg} \mathrm{ha}^{-1}\right)\end{array}$ & $\begin{array}{c}\mathrm{Ca} \\
(\mathrm{Cmol} \\
\left.\left(\mathrm{p}^{+}\right) \mathrm{kg}^{-1}\right)\end{array}$ & $\begin{array}{c}\mathrm{Mg} \\
\left(\mathrm{Cmol}^{+}\right. \\
\left.\left(\mathrm{p}^{+}\right) \mathrm{kg}^{-1}\right)\end{array}$ & $\begin{array}{c}\mathrm{S} \\
\left(\mathrm{mg} \mathrm{kg}^{-1}\right)\end{array}$ \\
\hline 1 & 29 & $\begin{array}{c}183.02-231.19 \\
(212.68)\end{array}$ & $\begin{array}{c}1.36-10.87 \\
(7.07)\end{array}$ & $\begin{array}{c}408.0-448.0 \\
(432.38)\end{array}$ & $\begin{array}{c}16.3-26.1 \\
(22.51)\end{array}$ & $\begin{array}{c}14.26-23.98 \\
(18.14)\end{array}$ & $\begin{array}{c}5.89-6.12 \\
(5.97)\end{array}$ \\
\hline 2 & 27 & $\begin{array}{c}274.54-288.99 \\
(281.76)\end{array}$ & $\begin{array}{c}6.76-8.67 \\
(7.71)\end{array}$ & $\begin{array}{c}677.6-706.72 \\
(692.16)\end{array}$ & $\begin{array}{c}21.4-44.7 \\
(33.05)\end{array}$ & $\begin{array}{c}17.48-24.69 \\
(21.08)\end{array}$ & $\begin{array}{c}6.16-7.47 \\
(6.81)\end{array}$ \\
\hline 3 & 22 & $\begin{array}{c}197.47-279.35 \\
(232.39)\end{array}$ & $\begin{array}{c}5.89-7.3 \\
(6.71)\end{array}$ & $\begin{array}{c}520.8-801.92 \\
(630.56)\end{array}$ & $\begin{array}{c}22.5-30.4 \\
(27.77)\end{array}$ & $\begin{array}{c}11.78-20.97 \\
(16.29)\end{array}$ & $\begin{array}{c}5.96-10.68 \\
(9.20)\end{array}$ \\
\hline 4 & 23 & $\begin{array}{c}168.57-298.62 \\
(223.96)\end{array}$ & $\begin{array}{c}3.77-7.3 \\
(5.60)\end{array}$ & $\begin{array}{c}514.08-595.84 \\
(544.04)\end{array}$ & $\begin{array}{c}21.09-25.09 \\
(23.21)\end{array}$ & $\begin{array}{c}16.35-21.34 \\
(18.59)\end{array}$ & $\begin{array}{c}6.3-9.12 \\
(7.10)\end{array}$ \\
\hline 5 & 25 & $124.08-236.0$ & $2.98--7.71$ & $479.36-680.96$ & $12.5-21.1$ & $10.29-20.55$ & $5.83-7.02$ \\
$(197.09)$ & $(5.76)$ & $(591.73)$ & $(16.39)$ & $(14.08)$ & $16.27)$ \\
\hline 6 & 26 & $144.49-284.17$ & $3.81-7.45$ & $544.32-750.4$ & $30.7-31.0$ & $16.14-22.34$ & $6.71-7.73$ \\
\hline
\end{tabular}


"Status of macro nutrients in some soils of Central Farm of MKV, Parbhani (Maharashtra)"

\begin{tabular}{|c|c|c|c|c|c|c|c|}
\hline & & $(214.33)$ & (5.63) & $(647.36)$ & $(30.85)$ & (19.24) & $(7.22)$ \\
\hline 7 & 28 & $\begin{array}{c}110.77-260.09 \\
(156.78)\end{array}$ & $\begin{array}{c}6.29-7.38 \\
(6.95)\end{array}$ & $\begin{array}{c}483.61-837.76 \\
(637.90) \\
\end{array}$ & $\begin{array}{c}16.5-26.9 \\
(22.25)\end{array}$ & $\begin{array}{c}7.5-23.68 \\
(13.76) \\
\end{array}$ & $\begin{array}{c}6.48-10.09 \\
(8.33) \\
\end{array}$ \\
\hline 8 & 30 & $\begin{array}{c}260.09-279.35 \\
(274.53)\end{array}$ & $\begin{array}{c}3.51-7.09 \\
(5.85)\end{array}$ & $\begin{array}{c}413.72-823.2 \\
(613.31)\end{array}$ & $\begin{array}{c}16.1-30.1 \\
(23.37)\end{array}$ & $\begin{array}{l}8.7-16.0 \\
(11.22)\end{array}$ & $\begin{array}{c}5.34-10.18 \\
(6.77)\end{array}$ \\
\hline 9 & 21 & $\begin{array}{c}158.94-207.11 \\
(178.20)\end{array}$ & $\begin{array}{c}3.25-4.23 \\
(3.63)\end{array}$ & $\begin{array}{c}560.0-753.76 \\
(660.09) \\
\end{array}$ & $\begin{array}{c}15.1-25.3 \\
(19.9)\end{array}$ & $\begin{array}{l}8.7-21.5 \\
(14.90) \\
\end{array}$ & $\begin{array}{c}6.4-7.7 \\
(7.07) \\
\end{array}$ \\
\hline 10 & $22 \mathrm{SB}$ & $\begin{array}{c}144.49-231.19 \\
(199.39)\end{array}$ & $\begin{array}{c}2.97-6.73 \\
(5.15)\end{array}$ & $\begin{array}{c}432.32-713.44 \\
(573.47)\end{array}$ & $\begin{array}{c}15.1-34.5 \\
(26.36)\end{array}$ & $\begin{array}{c}12.3-24.3 \\
(18.20)\end{array}$ & $\begin{array}{c}6.36-10.29 \\
(7.74)\end{array}$ \\
\hline 11 & $23 \mathrm{SB}$ & $\begin{array}{c}38.53-327.52 \\
(211.25)\end{array}$ & $\begin{array}{c}2.76-9.11 \\
(5.64)\end{array}$ & $\begin{array}{c}450.5-711.2 \\
(541.12)\end{array}$ & $\begin{array}{c}11.9-44.7 \\
(23.28)\end{array}$ & $\begin{array}{c}15.3-18.3 \\
(16.98)\end{array}$ & $\begin{array}{c}5.51-9.9 \\
(7.25)\end{array}$ \\
\hline 12 & $24 \mathrm{SB}$ & $\begin{array}{c}158.94-236.0 \\
(205.90) \\
\end{array}$ & $\begin{array}{c}5.17-6.83 \\
(6.22)\end{array}$ & $\begin{array}{c}461.44-635.04 \\
(549.64) \\
\end{array}$ & $\begin{array}{c}18.2-34.3 \\
(26.37) \\
\end{array}$ & $\begin{array}{c}11.9-22.5 \\
(17.45) \\
\end{array}$ & $\begin{array}{c}5.68-9.56 \\
(7.31) \\
\end{array}$ \\
\hline 13 & 24 & $\begin{array}{c}86.69-274.54 \\
(169.28) \\
\end{array}$ & $\begin{array}{c}5.12-8.02 \\
(6.98)\end{array}$ & $\begin{array}{c}452.48-826.56 \\
(601.62)\end{array}$ & $\begin{array}{c}24.4-32.2 \\
(28.73)\end{array}$ & $\begin{array}{c}18.4-25.4 \\
(21.43) \\
\end{array}$ & $\begin{array}{c}4.51-9.95 \\
(6.34) \\
\end{array}$ \\
\hline 14 & $27 \mathrm{SB}$ & $\begin{array}{c}96.33-308.25 \\
(200.36)\end{array}$ & $\begin{array}{c}4.51-7.78 \\
(6.01)\end{array}$ & $\begin{array}{c}483.61-750.4 \\
(607.21)\end{array}$ & $\begin{array}{c}18.7-39.5 \\
(28.88) \\
\end{array}$ & $\begin{array}{c}12.2-25.9 \\
(20.24) \\
\end{array}$ & $\begin{array}{c}5.98-9.33 \\
(7.57) \\
\end{array}$ \\
\hline 15 & $\begin{array}{c}25 / 26 \\
\text { SB }\end{array}$ & $\begin{array}{c}62.61-317.88 \\
(214.32)\end{array}$ & $\begin{array}{c}2.55-7.97 \\
(4.86)\end{array}$ & $\begin{array}{c}481.6-747.04 \\
(610.82)\end{array}$ & $\begin{array}{c}29.1-39.0 \\
(34.17)\end{array}$ & $\begin{array}{c}11.2-28.5 \\
(18.75)\end{array}$ & $\begin{array}{c}5.96-9.96 \\
(8.14)\end{array}$ \\
\hline 16 & 8 & $\begin{array}{c}144.49-298.62 \\
(216.74)\end{array}$ & $\begin{array}{c}2.78-4.3 \\
(3.44) \\
\end{array}$ & $\begin{array}{c}514.08-750.4 \\
(620.10) \\
\end{array}$ & $\begin{array}{c}23.6-36.8 \\
(30.76) \\
\end{array}$ & $\begin{array}{c}18.5-24.0 \\
(21.30) \\
\end{array}$ & $\begin{array}{c}9.54-9.84 \\
(9.69) \\
\end{array}$ \\
\hline 17 & 9 & $\begin{array}{c}86.69-298.62 \\
(209.99) \\
\end{array}$ & $\begin{array}{c}2.38-10.97 \\
(5.30)\end{array}$ & $\begin{array}{c}544.32-826.56 \\
(725.53) \\
\end{array}$ & $\begin{array}{c}24.7-56.1 \\
(38.98) \\
\end{array}$ & $\begin{array}{c}14.4-35.9 \\
(24.20) \\
\end{array}$ & $\begin{array}{c}6.56-9.08 \\
(7.56) \\
\end{array}$ \\
\hline 18 & 10 & $\begin{array}{c}183.02-248.21 \\
(212.27)\end{array}$ & $\begin{array}{c}2.78-4.28 \\
(3.84)\end{array}$ & $\begin{array}{c}595.84-762.72 \\
(686.56) \\
\end{array}$ & $\begin{array}{c}19.2-36.3 \\
(28.15) \\
\end{array}$ & $\begin{array}{c}11.2-21.1 \\
(16.55) \\
\end{array}$ & $\begin{array}{c}5.97-7.14 \\
(6.69) \\
\end{array}$ \\
\hline 19 & 20 & $\begin{array}{c}62.61-255.27 \\
(149.30)\end{array}$ & $\begin{array}{c}2.95-6.12 \\
(3.93)\end{array}$ & $\begin{array}{c}502.88-731.36 \\
(617.40)\end{array}$ & $\begin{array}{c}27.5-34.1 \\
(31.75)\end{array}$ & $\begin{array}{c}16.0-34.8 \\
(21.95)\end{array}$ & $\begin{array}{c}6.77-9.62 \\
(7.99)\end{array}$ \\
\hline 20 & 16 & $\begin{array}{c}110.77-274.54 \\
(208.31)\end{array}$ & $\begin{array}{c}4.15-5.58 \\
(5.04)\end{array}$ & $\begin{array}{c}\text { 655.2-819.84 } \\
(762.44)\end{array}$ & $\begin{array}{c}24.8-34.9 \\
(28.70)\end{array}$ & $\begin{array}{c}12.1-20.0 \\
(15.90)\end{array}$ & $\begin{array}{c}5.04-6.9 \\
(6.28)\end{array}$ \\
\hline 21 & 37 & $\begin{array}{c}158.94-351.6 \\
(239.61)\end{array}$ & $\begin{array}{c}3.18-6.66 \\
(5.05)\end{array}$ & $\begin{array}{c}617.12-879.2 \\
(748.44)\end{array}$ & $\begin{array}{c}16.5-34.5 \\
(26.30)\end{array}$ & $\begin{array}{l}9.4-19.7 \\
(16.35) \\
\end{array}$ & $\begin{array}{c}5.86-6.86 \\
(6.33)\end{array}$ \\
\hline 22 & 17 & $\begin{array}{c}77.06-211.92 \\
(138.71) \\
\end{array}$ & $\begin{array}{c}4.12-7.23 \\
(5.35)\end{array}$ & $\begin{array}{c}404.32-575.2 \\
(492.70) \\
\end{array}$ & $\begin{array}{c}15.1-30.3 \\
(22.12)\end{array}$ & $\begin{array}{l}8.7-16.6 \\
(12.20) \\
\end{array}$ & $\begin{array}{c}5.75-7.9 \\
(7.16) \\
\end{array}$ \\
\hline 23 & 18 & $\begin{array}{c}110.77-255.27 \\
(167.60)\end{array}$ & $\begin{array}{l}5.9-10.48 \\
(7.41)\end{array}$ & $\begin{array}{c}395.36-578.56 \\
(462.52)\end{array}$ & $\begin{array}{c}20.2-36.8 \\
(29.78)\end{array}$ & $\begin{array}{l}11.6-21 \\
(16.66)\end{array}$ & $\begin{array}{c}6.67-9.64 \\
(7.62)\end{array}$ \\
\hline 24 & 19 & $\begin{array}{c}227.52-255.27 \\
(241.39)\end{array}$ & $\begin{array}{c}3.82-5.32 \\
(4.57)\end{array}$ & $\begin{array}{c}445.76-449.12 \\
(447.44)\end{array}$ & $\begin{array}{c}23.0-28.5 \\
(25.75)\end{array}$ & $\begin{array}{c}14.9-18.5 \\
(16.70)\end{array}$ & $8.3-9 .(8.69)$ \\
\hline
\end{tabular}

The results of study presented in Table1 indicated that in all the soil samples of Central Farm - B Block of MKV, Parbhani, the available N was found low to medium (38.53 to $351.60 \mathrm{~kg} \mathrm{ha}^{-1}$ ) in soils of Central Farm - B - Block of MKV, Parbhani. It may be due difference in organic matter content of soil (Malewar et.al. 1998).The soils of Central Farm - B - Block of MKV, Parbhani were found low to medium in available P content. The variation in the availability of phosphorus might be due to variation in $\mathrm{CaCO}_{3}$ content in the soil, different soil properties and agronomic practices. The similar results were also reported by Bharambe et al. (1999) In case of available K the soils of Central Farm - B - Block of MKV, Parbhani were found sufficiently supplied with available K. This may be due to occurrence of potash rich minerals like mica and feldspar the available K was high Malewar (1995) and More et al (2013).

In case of secondary nutrients, the exchangeable Ca content in the soils of Central Farm - B - Block of MKV, Parbhani (11.90 to $56.10 \mathrm{C} \mathrm{mol}\left(\mathrm{p}^{+}\right) \mathrm{kg}^{-1}$ ) were found sufficient. While the exchangeable Mg was also found in sufficient amount in Central Farm - B - Block of MKV, Parbhani (7.5 to $\left.35.90 \mathrm{Cmol}\left(\mathrm{p}^{+}\right) \mathrm{kg}^{-1}\right)$. The available $\mathrm{S}$ was found low to medium in soils of Central Farm - B - Block of MKV, Parbhani (4.51 to $10.68 \mathrm{mg}$ $\left.\mathrm{kg}^{-1}\right)$. This could be attributed due to high amount of clay content in soils which can adsorbed varying amounts of S. This might be expected due to presence of Fe and Al oxides in surface soils Singh et al. (2006). Summarizing the results, it can be concluded that the soils of - B - block were neutral to alkaline in soil reaction, safe in electrical conductivity, low to high in organic carbon content and non-calcareous to calcareous in nature.

\section{References}

[1]. Bharambe, P.R. Kadam, S. G.Shinde, S.D. and Shelke, D.K. (1999). Characterization of soils soils Majalgaon canal command area (Jayakwadi project stage II) J. Indian Soc. Soil Sci.47 (4) : 749-754

[2]. Jackson, M.L. (1973). Soil Chemical analysis Prentice hall of Indian Private Ltd. New Delhi.

[3]. Landy, M.G., Varade, S. B. and Badhe, N. N. (1977). Sulphur status and its relationship with physcio-chemical properties of Marathwada soils. J. Maharashtra Agric Univ 2 (3): 195-201.

[4]. Malewar, G.U., Dhamak, A.L., and Syed Ismail (1998). Inter-relationship f forms of Fe with properties of soils of semi-arid area of Northern Marathwada. J. Maharashrta Agric Univ., 23 (2): 199-201.

[5]. Malewar, G.U. (1995). Micronutrient availability as influenced by cropping pattern in Marathwada region of Maharashtra. J. Maharashtra Agric. Univ. 20 (3): 330-333. 
[6]. Meena, H.B., Sharma, R.P. and. Rawat, U.S (2006). Status of macro and micronutrients in some soils of Tonk district of Rajasthan Journal of the Indian Society of Soil Science, 54 (4):508-512.

[7]. More,S.S. Waikar,S.L. and S.E. Shinde (2013) Macronutrient status of soils from Basmat tahsil in Hingoli district.Green Farming Int.J, 4 (4):443-445.

[8]. Padole, V.R. and Mahajan, S.B. (2001). Potassium fixation characteristics of swell-shrink soils of Vidarbha. (Maharashtra). J. Maharashtra Agric. Univ. 25 (2): 134-138.

[9]. Singh, A.H., Singh,K L. N., N. Gopimohan Singh, Nandini Chongtham, and Kumar Singh (2006). Status and forms of sulphur in acidic soils of Manipur. J. Indian Soc. Soil Sci. 54(3): 351-353.

[10]. Subbaih, B. V. and Asija, G. L. (1956). Rapid procedure for the estimation of available nitrogen in soil Curr. Sci. 125: 259-260.
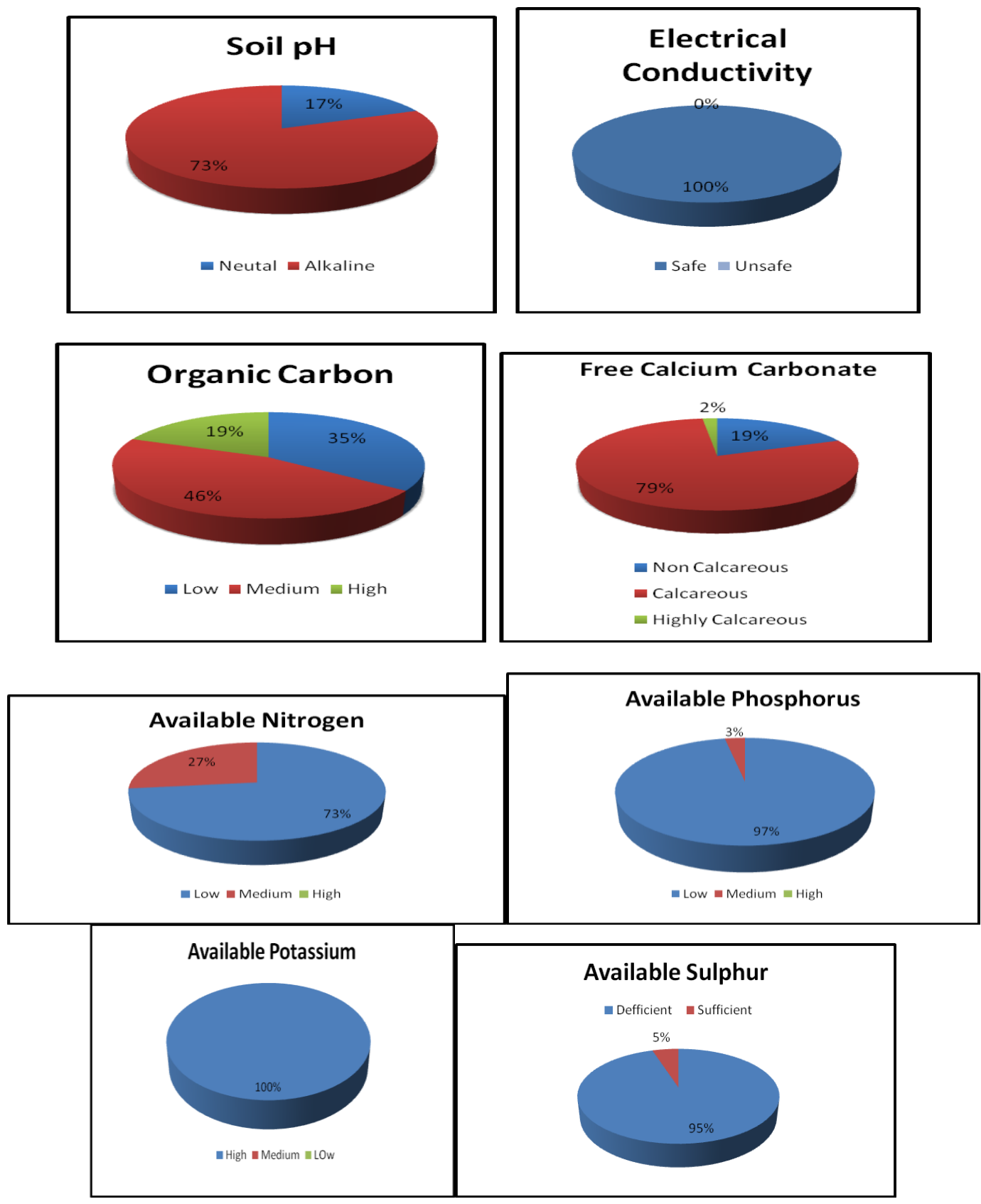

Fig: 1 Chemical Properties of soils. 